Draft VERSion MAY 21, 2018

Preprint typeset using $\mathrm{LAT}_{\mathrm{E}} \mathrm{X}$ style emulateapj v. 08/22/09

\title{
THE BIRTH OF A GALAXY: PRIMORDIAL METAL ENRICHMENT AND STELLAR POPULATIONS
}

\author{
John H. Wise ${ }^{1}$, Matthew J. Turk ${ }^{2}$, Michael L. Norman ${ }^{3}$, Tom Abel ${ }^{4,5,6}$ \\ Draft version May 21, 2018
}

\begin{abstract}
By definition, Population III stars are metal-free, and their protostellar collapse is driven by molecular hydrogen cooling in the gas-phase, leading to large characteristic masses. Population II stars with lower characteristic masses form when the star-forming gas reaches a critical metallicity of $10^{-6}-10^{-3.5} Z_{\odot}$. We present an adaptive mesh refinement radiation hydrodynamics simulation that follows the transition from Population III to II star formation. The maximum spatial resolution of 1 comoving parsec allows for individual molecular clouds to be well-resolved and their stellar associations to be studied in detail. We model stellar radiative feedback with adaptive ray tracing. A top-heavy initial mass function for the Population III stars is considered, resulting in a plausible distribution of pair-instability supernovae and associated metal enrichment. We find that the gas fraction recovers from 5 percent to nearly the cosmic fraction in halos with merger histories rich in halos above $10^{7} M_{\odot}$. A single pair-instability supernova is sufficient to enrich the host halo to a metallicity floor of $10^{-3} Z_{\odot}$ and to transition to Population II star formation. This provides a natural explanation for the observed floor on damped Lyman alpha (DLA) systems metallicities reported in the literature, which is of this order. We find that stellar metallicities do not necessarily trace stellar ages, as mergers of halos with established stellar populations can create superpositions of $t-Z$ evolutionary tracks. A bimodal metallicity distribution is created after a starburst occurs when the halo can cool efficiently through atomic line cooling.

Subject headings: cosmology — methods: numerical — hydrodynamics — radiative transfer — star formation
\end{abstract}

\section{MOTIVATION}

The first (Pop III) stars are metal-free and have a large characteristic mass. Previously, these stars were believed to be massive, with universally suppressed fragmentation (Abel et al. 2002; Bromm et al. 2002; O’Shea \& Norman 2007). Recently, several studies have suggested that while fragmentation at times prior to the formation of the first protostar may occur at scales of $1000-2000 \mathrm{AU}$, such fragmentation is likely to result in high-mass, low-multiplicity groups (Turk et al. 2009; Stacy et al. 2010). Subsequent studies have suggested that at later times, subsequent to the formation of the first protostar, additional fragmentation may proceed (Greif et al. 2011a; Clark et al. 2011). A fraction of high-mass, metal-free stars enrich the surrounding intergalactic medium (IGM) when they go supernova, which can happen in stars $\lesssim 40 M_{\odot}$ in Type II supernovae (SNe) or in stars roughly between $140 M_{\odot}$ and 260 $M_{\odot}$ in pair-instability SNe (PISNe; Heger \& Woosley 2002a). The host halo and the neighboring halos are then enriched with this ejecta. There exists a critical

\footnotetext{
Electronic address: jwise@physics.gatech.edu

${ }^{1}$ Center for Relativistic Astrophysics, School of Physics, Georgia Institute of Technology, 837 State Street, Atlanta, GA 30332

2 Department of Astronomy, Columbia University, 538 West 120th Street, New York, NY 10027

${ }^{3}$ Center for Astrophysics and Space Sciences, University of California at San Diego, La Jolla, CA 92093

${ }^{4}$ Kavli Institute for Particle Astrophysics and Cosmology, Stanford University, Menlo Park, CA 94025

${ }^{5}$ Zentrum für Astronomie der Universität, Institut für Theoretische Astrophysik, Albert-Ueberle-Str. 2, 69120 Heidelberg, Germany

${ }^{6}$ Heidelberg Institut für Theoretische Studien, SchlossWolfsbrunnenweg 35, 69118 Heidelberg, Germany
}

metallicity that is $\sim 10^{-6} Z_{\odot}$ if dust cooling is efficient Omukai et al. 2005; Schneider et al. 2006; Clark et al. 2008) and $\sim 10^{-3.5} Z_{\odot}$ otherwise (Bromm et al. 2001; Smith et al. 2009), where the gas can cool rapidly, lowering its Jeans mass. An intermediate characteristic mass of $\sim 10 M_{\odot}$ can occur if the gas cooling is suppressed to the cosmic microwave background (CMB) temperature (Larson 1998; Tumlinson 2007; Smith et al. 2009). The resulting Population II star cluster will thus have a lower characteristic stellar mass than its metal-free progenitors. These first stellar clusters may be connected to stars in the Milky Way halo and nearby dwarf spheroidal (dSph) galaxies, both with a metallicity floor of $[\mathrm{Z} / \mathrm{H}]^{7}=-4$ (Beers \& Christlieb 2005; Frebel et al. 2010; Tafelmever et al. 2010).

Pop III stars primarily form in dark matter halos with $M \sim 10^{6} M_{\odot}$. Their stellar radiation ionizes and heats an $\mathrm{H}$ II region with a radius $1-3 \mathrm{kpc}$. The over-pressurized $\mathrm{H}$ II region drives a $30 \mathrm{~km} \mathrm{~s}^{-1}$ shock that is 10 times greater than the escape velocity of the halo, leaving behind a warm $\left(3 \times 10^{4} \mathrm{~K}\right)$ and diffuse $\left(0.5 \mathrm{~cm}^{-3}\right)$ medium (Kitavama et al. 2004; Whalen et al. 2004; Abel et al. 2007). This aids in the expansion of the blastwave because it delays the transition to the Sedov-Taylor and snowplow phases. In PISNe, approximately half of the metals stay in the IGM with a metal bubble size of a few kpc (Wise \& Abel 2008; Greif et al. 2010). The blastwave may induce star formation (SF) in nearby halos through the compression of the gas (Ferrara 1998), and timescales for metal mixing into the dense gas are many dynamical times (Cen \& Riquelme 2008) for shock veloc-

7 We use the conventional notation, $[\mathrm{Z} / \mathrm{H}] \equiv \log (\mathrm{Z} / \mathrm{H})-$ $\log \left(\mathrm{Z}_{\odot} / \mathrm{H}_{\odot}\right)$. 
ities $\lesssim 100 \mathrm{~km} \mathrm{~s}^{-1}$.

The transition from Pop III to Pop II SF is solely dependent on the propagation of metals from the SNe remnants into future sites of SF. Their flows are complex because of the interactions between the SN blastwave, cosmological accretion, halo mergers, and nearby stellar feedback. Spurred by the notion of a critical metallicity, this transition has been extensively studied with (i) volumeaveraged semi-analytic models (Scannapieco et al. 2003; Yoshida et al. 2004; Furlanetto \& Loeb 2005), (ii) models using hierarchical merger trees (Tumlinson 2006, 2010; Salvadori et al. 2007; Komiva et al. 2010), (iii) post-processing of cosmological simulations with blastwave models (Karlsson et al. 2008; Trenti et al. 2009; Trenti \& Shull 2010), and (iv) direct numerical simulations with stellar feedback (Tornatore et al. 2007; Ricotti et al. 2008; Maio et al. 2010, 2011).

Over the past decade, these works have refined the general picture of inhomogeneous metal enrichment and the transition to Pop II star formation, and here we give an overview of its development. Considering only Pop III star formation, Yoshida et al. calculated that Pop III stars can raise the mean metallicity to $-4.5 \lesssim$ $[\mathrm{Z} / \mathrm{H}] \lesssim-3.5$ by redshift 15 in the upper limit where all metal-free stars have $M=200 M_{\odot}$ and produce PISNe. Considering both Pop III and II star formation, Scannapieco et al. found that the transition between the two modes is a gradual process where both modes are coeval, confirmed by most of the later works. Because the host galaxies have small masses and are subject to negative radiative feedback through photo-heating and photo-dissociation, the minimum halo mass gradually increases with the radiation background intensity, which Furlanetto \& Loeb found to delay metal enrichment and place it closer to the epoch of reionization. Trenti et al. noted that underdense regions of the universe are still pristine at $z=6$ with Pop III stars still forming at these late epochs. This group later expanded on these results to find that Pop II SFR becomes dominant at $z<25$ in the buildup of a Milky Way (MW) like halo. Furthermore they stress the importance of a photo-dissociating radiation background that reduces enrichment by PISNe and increases the importance of metal-rich galactic outflows, where only $10^{-4}-10^{-2}$ of PISN ejecta ends up in extremely metal poor (EMP) stars with $M>0.9 M_{\odot}$.

The Pop III IMF should play an important role in abundance patterns in EMP stars in the MW halo and the physics of reionization. Using these data as constraints, Tumlinson found that Pop III IMFs with lognormal distributions with mean masses between 8 and $42 M_{\odot}$ best fit the data. Furthermore he concludes that EMP stars with $[\mathrm{Z} / \mathrm{H}]<-3$ have between 1 and 10 metalfree SN progenitors and the Pop III SFR is less than $1 \%$ of the total SFR at $z=6$. Karlsson et al. use observational data of EMP stars with their model to constrain the mass fraction of Pop III stars that die with a PISN is less than 40 per cent. They also conclude that stars enriched primarily by PISNe have $[\mathrm{Ca} / \mathrm{H}] \gtrsim-2.6$, which could have been missed by some EMP surveys.

These works have clearly shown that many processes play a key role in the transition from Pop III to II stars, but how does their interplay affect the formation of the first galaxies? Will metal-free pockets of gas still exist in dwarf galaxies that are already forming metal-enriched stars? How do the metal-enriched ejecta propagate into future sites of star formation? Numerical simulations are powerful tools to detangle and study these complexities. To answer such questions, we have carried out a simulation that includes both types of SF and their radiative and mechanical feedback. The methods used here incorporate and link together recent results from metal-enriched and metal-free star formation, the critical metallicity, and pair-instability supernovae. This is the first time it has been possible to correlate the formation and feedback of the first stars to protogalaxies, resolving individual molecular clouds and minihalos down to $10^{5} M_{\odot}$ and including the most important physical effects, such as radiative and supernova feedback.

We first describe the simulation setup and numerical methods behind our work in $\$ 2$ Then in 93 we present the evolution of two selected high-redshift dwarf galaxies, focusing on their metallicities and stellar populations. Last we discuss possible dependencies of our results on the Pop III initial mass function and conclude in $\$ 4$.

\section{METHOD}

\subsection{Simulation setup}

We use the adaptive mesh refinement (AMR) code Enzo v2.0 ${ }^{8}$ (O'Shea et al. 2004). It uses an $N$-body adaptive particle-mesh solver (Efstathiou et al. 1985; Couchman 1991; Bryan \& Norman 1997) to follow the dark matter (DM) dynamics. It solves the hydrodynamical equation using the second-order accurate piecewise parabolic method (Woodward \& Colella 1984; Bryan et al. 1995), while a Riemann solver ensures accurate shock capturing with minimal viscosity. We use the recently added HLLC Riemann solver (Toro et al. 1994) for additional stability in strong shocks and rarefaction waves. We use the nine-species (H I, H II, He I, He II, He III, $\left.\mathrm{e}^{-}, \mathrm{H}_{2}, \mathrm{H}_{2}{ }^{+}, \mathrm{H}^{-}\right)$non-equilibrium chemistry model in Enzo (Abel et al. 1997; Anninos et al. 1997) and the $\mathrm{H}_{2}$ cooling rates from Glover \& Abel (2008). We spatially distinguish metal enrichment from Pop II and Pop III stars. We will follow-up this study with one that considers radiative cooling from metal-enriched gas, using the method of Smith et al. (2008) that is already implemented in Enzo.

To resolve minihalos with at least 100 dark matter (DM) particles and follow the formation of the first generation of dwarf galaxies, we use a simulation box of $1 \mathrm{Mpc}$ that has a resolution of $256^{3}$. This gives us a DM mass resolution of $1840 M_{\odot}$. We refine the grid on baryon overdensities of $3 \times 2^{-0.2 l}$, where $l$ is the AMR level, resulting in a super-Lagrangian behavior (also see O'Shea \& Norman 2008). We also refine on a DM overdensity of three and always resolve the local Jeans length by at least 4 cells, avoiding artificial fragmentation during gaseous collapses (Truelove et al. 1997). If any of these criteria are met in a single cell, it is flagged for further spatial refinement.

We initialize the simulation with grafic Bertschinger 2001) at $z=130$ and use the cosmological parameters from the 7-year WMAP $\Lambda \mathrm{CDM}+\mathrm{SZ}+\mathrm{LENS}$ best

\footnotetext{
8 enzo.googlecode.com, changeset b86d8ba026d6
} 
fit (Komatsu et al. 2011): $\Omega_{M}=0.266, \Omega_{\Lambda}=0.734$, $\Omega_{b}=0.0449, h=0.71, \sigma_{8}=0.81$, and $n=0.963$ with the variables having their usual definitions. We use a maximum refinement level of $l=12$, resulting in a maximal comoving resolution of 1 pc. The adaptive particle-mesh solver has a force resolution of two cell widths of a given AMR grid. We stop the simulation at $z=7$ to prevent any large-scale modes with $r \sim L_{\text {box }} / 2$ from entering the non-linear growth regime. At the final redshift, the simulation has $1.4 \times 10^{8}$ computational cells and required 250,000 CPU hours, using 512 cores.

\subsection{Star formation}

We distinguish Pop II and Pop III SF by the total metallicity of the densest cell in the molecular cloud. Pop II stars are formed if $[\mathrm{Z} / \mathrm{H}]>-4$, and Pop III stars are formed otherwise. We do not consider Pop III.2 stars and intermediate mass stars from CMB-limited cooling.

\subsubsection{Population III star formation}

Simulations following the full accretion history of Pop III stars, from their formation inside cosmological minihalos through transitioning onto the main sequence, are not currently possible. However, under the assumption of spherical accretion, reasonable estimates of the mass ranges for Pop III stars can be made by extrapolating forward in time the instantaneous accretion rate at the time of formation of the first protostellar core and comparing the timescales for accretion against the Kelvin-Helmholtz time. Several studies, conducting this extrapolation, have shown that the characteristic mass of Pop III stars $M_{\text {char }} \sim 100 M_{\odot}$ (Abel et al. 2002; Bromm et al. 2002; O'Shea \& Norman 2007; Yoshida et al. 2008). They form in molecular clouds that coexist with the dark matter halo center with limited fragmentation occurring during their collapse; however Turk et al. (2009) and Stacy et al. (2010) have shown that Pop III binaries may form in a fraction of such halos. There have been some recent hints that the accretion disk around the first protostar may fragment and produce clustered Pop III star formation (Clark et al. 2011; Greif et al. 2011a). The final stellar masses in the aforementioned calculations are still highly uncertain with a limited number of samples. Nevertheless, they all point toward an IMF with a larger characteristic mass than present-day star formation.

For Pop III stars, we use the same SF model as Wise \& Abel (2008) where each star particle represents a single star. In this model, a star particle forms when a cell has all of the following criteria:

1. an overdensity of $5 \times 10^{5}\left(\sim 10^{3} \mathrm{~cm}^{-3}\right.$ at $\left.z=10\right)$,

2. a converging gas flow $\left(\nabla \cdot \mathbf{v}_{\text {gas }}<0\right)$, and

3. a molecular hydrogen fraction $f_{\mathrm{H} 2}>5 \times 10^{-4}$.

These physical conditions are typical of collapsing metalfree molecular clouds $\sim 10 \mathrm{Myr}$ before the birth of a Pop III main-sequence star Abel et al. 2002; O'Shea \& Norman 2007). This prescription is similar to the Cen \& Ostriker (1992) method but removes the Jeans unstable $\left(M_{\text {gas }}>M_{J}\right)$ and cooling timescale $\left(t_{\text {cool }}<t_{\text {dyn }}\right)$ requirements. We do not consider the former criterion because it is not applicable to simulations that resolve the Jeans length at all times. The molecular hydrogen fraction requirement effectively constrains star formation to cooling molecular clouds, where the $\mathrm{H}_{2}$ formation rate is significantly larger than the dissociation rate from a Lyman-Werner radiation field.

If multiple cells meet the star particle formation criteria within $1 \mathrm{pc}$, we form one Pop III star particle with the center of mass of these flagged cells to ensure that one massive star is created per metal-free molecular cloud. Instead of using a fixed stellar mass like in our previous work, we randomly sample from an IMF with a functional form of

$$
f(\log M) d M=M^{-1.3} \exp \left[-\left(\frac{M_{\text {char }}}{M}\right)^{1.6}\right] d M
$$

to determine the stellar mass. Above $M_{\text {char, it }}$ behaves as a Salpeter IMF but is exponentially cutoff below that mass (Chabrier 2003; Clark et al. 2009). For reproducibility, we record the number of times the random number generator (Mersenne twister; Matsumoto \& Nishimura (1998)) has been called for use when restarting the simulations. We stress that the Pop III IMF is still highly uncertain. In Sec. 4, we discuss possible dependencies of our results to changes in the IMF.

After the star particle forms and its mass is randomly sampled from the IMF, an equal amount of gas is removed from the computational grid in a sphere that contains twice the stellar mass and is centered on the star particle. The star particle acquires the mass-weighted velocity of the gas contained in this sphere.

\subsubsection{Population II star formation}

We treat Pop II star formation with the same prescription as Wise \& Cen (2009), which is similar to the Pop III prescription but without the minimum $\mathrm{H}_{2}$ fraction requirement. This is removed because the metal-enriched gas can efficiently cool even in the presence of a strong UV radiation field (e.g. Safranek-Shrader et al. 2010). To ensure the volume is cooling, we restrict star formation to gas with temperatures $T<1000 \mathrm{~K}$. Unlike Pop III star particles that represent individual stars, Pop II star particles represent a star cluster of some total mass and an assumed normal IMF.

Once a cell meets these criteria, the prescription searches outward with increasing radius for the boundary of the molecular cloud, centered on the most dense cell. Here, we define a molecular cloud as a sphere with a dynamical time $t_{\mathrm{dyn}}=3 \mathrm{Myr}$ (corresponding to an average density $\bar{\rho}_{\mathrm{MC}} \simeq 1000 \mu \mathrm{cm}^{-3}$ ) and a radius $R_{\mathrm{MC}}$, where $\mu$ is the mean molecular weight. Once the sphere radius is found, a fraction $c_{\star}=0.07 f_{\text {cold }}$ of the cold gas $\left(T<10^{3} \mathrm{~K}\right)$ inside the sphere is converted into a star particle with mass $m_{\star}=c_{\star}(4 \pi / 3) \bar{\rho}_{\mathrm{MC}} R_{\mathrm{MC}}^{3}$. This treatment of cold gas accretion is similar to a star formation model with a multi-phase subgrid model (Springel \& Hernquist 2003) but is employed in a simulation that can resolve the multi-phase interstellar stellar medium (ISM).

After the star particle is created, we replace the sphere with a uniform density $\rho_{\mathrm{MC}}=\left(1-c_{\star}\right) /\left(G t_{\mathrm{dyn}}^{2}\right)$ and temperature $T=10^{4} \mathrm{~K}$, which approximates the initial stages of an $\mathrm{H}$ II region. Our choice of $c_{\star}$ agrees with the 
theory proposed in Krumholz \& McKee (2005) that are supported by observations. Observations of star-forming regions show that the dynamical time is approximately $700 \mathrm{kyr}$ and that SF is extended over several dynamical times (e.g. Tan et al. 2006), which motivates our choice of $t_{\mathrm{dyn}}$ of our definition of molecular clouds in our SF prescription.

Similar to the Pop III treatment, we merge any newly created particles within a radius of $10 \mathrm{pc}$. The new star particle acquires the center of mass of the merged particles. During the merging, the gas is unaltered besides the initial gas accretion from the grid to the star particles. We set the minimum mass of a star particle to $m_{\star, \min }=1000 M_{\odot}$. If the initial mass does not exceed $m_{\star, \min }$, the star particle does not provide any feedback and continues to accrete until it reaches $m_{\star, \min }$.

\subsection{Stellar feedback}

The radiation field is evolved with adaptive ray tracing (Abel \& Wandelt 2002; Wise \& Abel 2011) that is based on the HEALPix framework (Górski et al. 2005) and is coupled self-consistently to the hydrodynamics. In this work, we only consider energy coupling to the gas; we defer an investigation of the effects of radiation momentum coupling to a subsequent paper. Each star particle is a point source of hydrogen ionizing radiation with the ionizing luminosity equally split between 48 initial rays (HEALPix level 2). We use a mono-chromatic spectrum for the radiation with the energy $E_{\mathrm{ph}}$ equaling the luminosity-weighted photon energy of the spectrum. For a cosmological simulation that focuses on galaxies, this does not significantly affect the overall galactic dynamics (see Sec. 6.3 in Wise \& Abel 2011). We do not consider helium singly- and doubly-ionizing radiation. As the rays propagate from the source or into a high resolution AMR grid, they are adaptively split into 4 child rays, increasing the angular resolution of the solution, when the solid angle of the ray $\theta=4 \pi /\left(12 \times 4^{L}\right)$ is larger than $1 / 3$ of the cell area, where $L$ is the HEALPix level. We model the $\mathrm{H}_{2}$ dissociating radiation with an optically-thin, inverse square profile, centered on all Pop II and III star particles. In principle, self-shielding effects can be included by utilizing our ray tracing framework or other approximate methods that calculate the $\mathrm{H}_{2}$ column density using the local Sobolev length (Wolcott-Green et al. 2011) or only integrating along Cartesian axes or random lines of sight (Yoshida et al. 2003, 2007; Glover \& Mac Low 2007). In halos with $M \gtrsim 10^{8} M_{\odot}$, the $\mathrm{H}_{2}$ column density may become large enough to self-shield itself from LW radiation (Wise \& Abel 2008). Thus we may be underestimating the SFRs in our work.

\subsubsection{Population III stellar feedback}

We use mass-dependent hydrogen ionizing and LW photon luminosities and lifetimes of the Pop III stars from Schaerer (2002). We use a mass-independent photon energy $E_{\mathrm{ph}}=29.6 \mathrm{eV}$, appropriate for the nearconstant $10^{5} \mathrm{~K}$ surface temperatures of Pop III stars. They die as Type II SNe if $11 \leq M_{\star} / M_{\odot} \leq 40$ $M_{\odot}$ (Woosley \& Weaver 1995) and as PISNe if they are in the mass range 140-260 $M_{\odot}$ (Heger et al. 2003), where $M_{\star}$ is the stellar mass. For normal Type II SNe between 11-20 $M_{\odot}$, we use an explosion energy of $10^{51} \mathrm{erg}$ and a linear fit to the metal ejecta mass calculated in Nomoto et al. (2006),

$$
M_{\mathrm{Z}} / M_{\odot}=0.1077+0.3383 \times\left(M_{\star} / M_{\odot}-11\right) .
$$

We model the SNe of stars with $20 \leq M_{\star} / M_{\odot} \leq 40$ as hypernova with the energies and ejecta masses also taken from Nomoto et al., linearly interpolating their results to $M_{\star}$. For PISNe, we use the explosion energy from Heger \& Woosley (2002b), where we fit the following function to their models,

$$
E_{\mathrm{PISN}}=10^{51} \times\left[5.0+1.304\left(M_{\mathrm{He}} / M_{\odot}-64\right)\right] \mathrm{erg},
$$

where $M_{\mathrm{He}}=(13 / 24) \times\left(M_{\star}-20\right) M_{\odot}$ is the helium core (and equivalently the metal ejecta) mass and $M_{\star}$ is the stellar mass. If the stellar mass is outside of these ranges, then an inert, collisionless black hole $(\mathrm{BH})$ particle is created. The blastwave is modeled by injecting the explosion energy and ejecta mass into a sphere of 10 pc, smoothed at its surface to improve numerical stability (Wise \& Abel 2008). Because we resolve the blastwave relatively well with several cells across at its initialization, the thermal energy is converted into kinetic energy and agrees with the Sedov-Taylor solution (e.g. Greif et al. 2007).

\subsubsection{Population II star formation}

The Pop II stars emit 6000 hydrogen ionizing photons per baryon over their lifetime and $E_{\mathrm{ph}}=21.6 \mathrm{eV}$, appropriate for a $[\mathrm{Z} / \mathrm{H}]=-1.3$ population. We note that lower-metallicity stars generate up to $60 \%$ more ionizing photons and might be underestimating the radiative feedback (Schaerer 2003). Nonetheless this study provides an excellent first insight in the transition to Pop II $\mathrm{SF}$, as the metal enrichment is the key ingredient here. The star particles live for $20 \mathrm{Myr}$, the maximum lifetime of an OB star. These stars generate the majority of the ionizing radiation and SNe feedback in stellar clusters, thus we ignore any feedback from lower mass stars.

For SN feedback, these stars generate $6.8 \times 10^{48} \mathrm{erg}$ $\mathrm{s}^{-1} M_{\odot}{ }^{-1}$ after living for $4 \mathrm{Myr}$, which is injected into spheres with a radius of $10 \mathrm{pc}$. However if the resolution of the grid is less than $10 / 3 \mathrm{pc}$, we distribute the energy into a $3^{3}$ cube surrounding the star particle. In the same region, star particles also return ejected material with mass

$$
\Delta m_{\mathrm{ej}}=\frac{0.25 \Delta t M_{\star}}{t_{0}-4 \mathrm{Myr}}
$$

back to the grid at every timestep on the finest AMR level. Here $t_{0}=20 \mathrm{Myr}$ is the lifetime of the star particle. This ejected gas has solar metallicity $Z=0.02$, resulting in a total metal yield $y=0.005$. In addition to numerical stability, energy distribution across several cells instead of a single cell has been shown to match cosmic SFRs better in galaxy simulations at lower redshifts (Smith et al. 2011).

\section{RESULTS}

Here we first present the global star formation rate (SFR) during early galaxy formation and then focus on the gaseous and stellar evolution of two selected halos in the simulation: one that has an early mass buildup but no major mergers after $z=12$, and one that experiences 


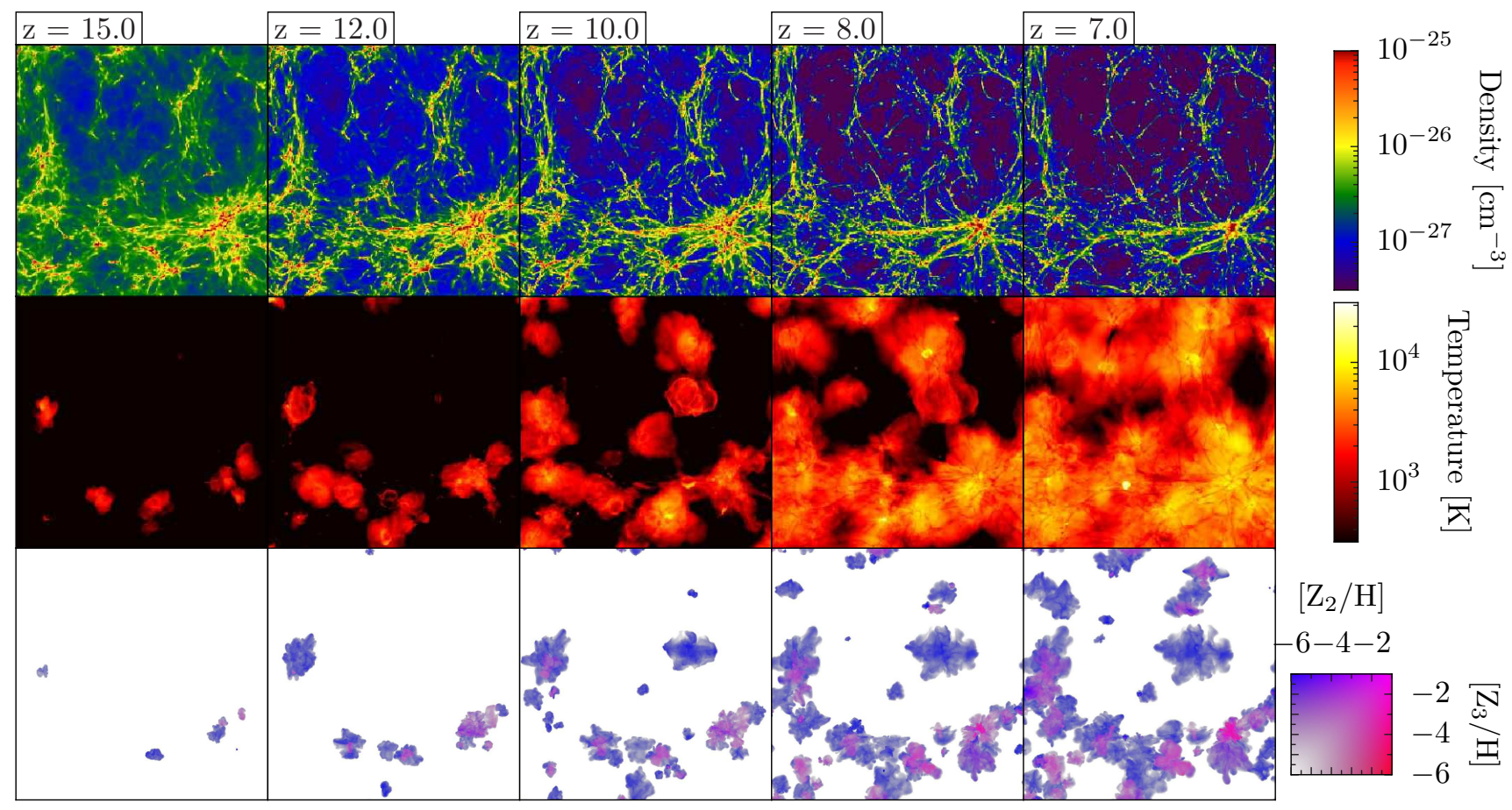

FIG. 1. - Evolution of the entire simulation volume $\left(L_{\mathrm{box}}=1 \mathrm{Mpc}\right)$ at redshifts $15,12,10,8$, and 7 (left to right). Pictured here are the density-weighted projections of density (top), temperature (middle), and metallicity (bottom). Note how the stellar radiative feedback from low-mass galaxies reionize the majority of the volume. The metallicity projections are a composite image of metals originating from Pop II (red) and III (blue) stars with magenta indicating a mixture of the two.

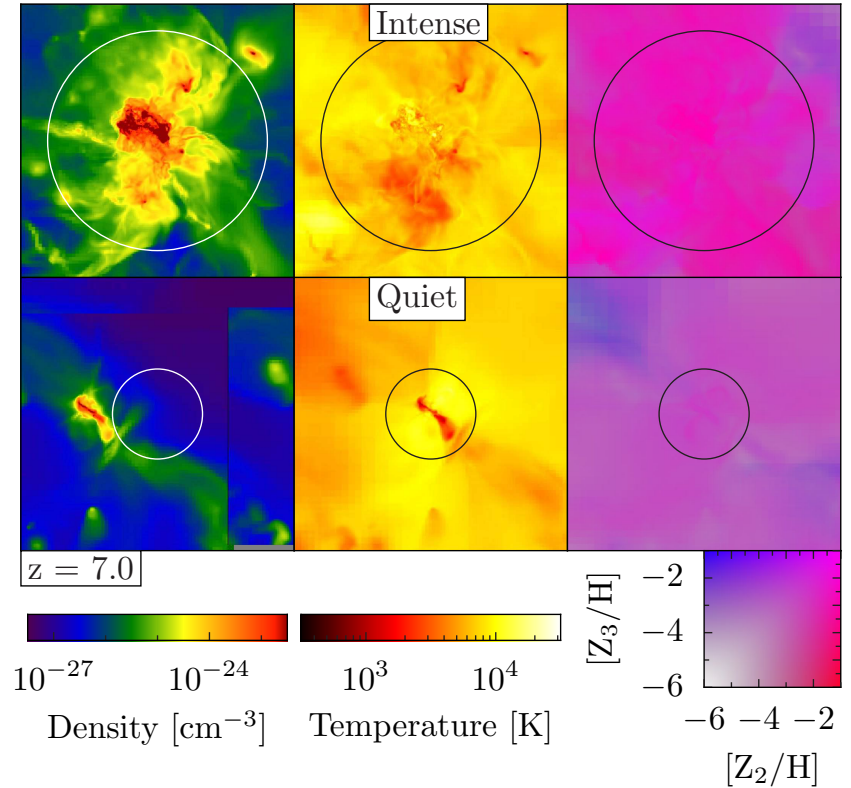

FIG. 2.- Density-weighted projections of density (left), temperature (center), and metallicity (right) of the two selected halos at $z=7$. The field of view is 5 proper $\mathrm{kpc}$, and the circles have a radius of $r_{200}$. The composite metallicity images are constructed in the same fashion as Fig. 1

a series of major mergers between $z=10$ and $z=7$. We name the halos "quiet" and "intense", respectively. The entire simulation contains 38 galaxies with 3640 Pop II stellar clusters and captures the formation of 333 Pop III stars.

We illustrate the evolution of the simulation from
TABLE 1

EnRICHMENT FraCtions at REDShift 7

\begin{tabular}{ccccc}
\hline \hline$[\mathrm{Z} / \mathrm{H}]$ & $f_{v}($ Pop III $)$ & $f_{m}($ Pop III $)$ & $f_{v}$ (Pop II $)$ & $f_{m}$ (Pop II) \\
\hline-6 & $3.81(-2)$ & $1.43(-1)$ & $5.52(-3)$ & $5.22(-2)$ \\
-5 & $3.28(-2)$ & $1.24(-1)$ & $3.92(-3)$ & $4.36(-2)$ \\
-4 & $2.65(-2)$ & $1.01(-1)$ & $2.33(-3)$ & $3.43(-2)$ \\
-3 & $1.86(-2)$ & $6.45(-2)$ & $1.00(-3)$ & $2.50(-2)$ \\
-2 & $8.29(-3)$ & $1.23(-2)$ & $2.61(-4)$ & $1.73(-2)$ \\
\hline
\end{tabular}

NoтE. $-f_{v}$ and $f_{m}$ are volume filling and mass-weighted fractions, respectively, above several different metallicity cuts. Values are listed in scientific notation with the exponents in parentheses.

$z=15$ to $z=7$ in Figure 1 with density weighted projections of gas density, temperature, and metallicity of the entire box. Figure 2 shows the same types of plots but focusing on the two halos of interest with a field of view of 5 proper kpc. Radiative and mechanical feedback create a multi-phase medium inside these halos, which are embedded in a warm and ionized IGM. At the final redshift, the volume and mass ionized fraction are 0.76 and 0.72 , respectively, which are consistent with largescale reionization simulations (Zahn et al. 2011). PISNe enrich 0.065 and 0.019 of the mass and volume, respectively, above $10^{-3} \mathrm{Z}_{\odot}$, and 0.025 and 0.0010 of the mass and volume are enriched by Type II SNe from Pop II stars above the same threshold. With $M_{\text {char }}=100 M_{\odot}$, a negligible fraction of Pop III stars die as Type II SNe; thus, all metals originating from Pop III stars are produced in PISNe. Table 1 gives additional enriched fractions with different metallicity cuts.

\subsection{Global star formation rate}




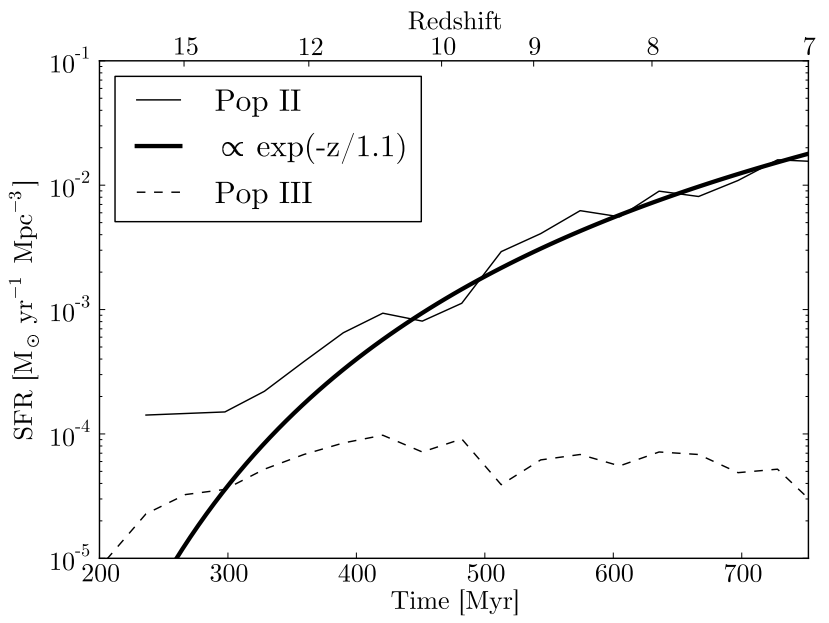

FIG. 3. - Cosmic star formation rates for Pop II (solid) and III (dashed). Note that the Pop III rates are nearly constant from redshift 12 to 7 . Pristine halos still survive in underdense regions, supporting late-time metal-free star formation.

Figure 3 shows the cosmic SFR in the simulation for Pop II and III stars. Metal-free stars form at a nearly constant rate of $5 \times 10^{-5} \mathrm{M}_{\odot} \mathrm{yr}^{-1} \mathrm{Mpc}^{-3}$ until the end of the simulation. The dissociating and ionization radiation from nearby Pop III stars and galaxies do not halt the formation of such stars in low-mass halos but only suppress them. We expect a uniform $\mathrm{H}_{2}$ dissociating radiation background to further delay them (Machacek et al. 2001; Wise \& Abel 2007; O'Shea \& Norman 2008).

The cosmic Pop II SFR is best fit with an exponential $\exp (-z / 1.1)$. Metal-enriched star formation begins early in the simulation at $z \sim 16$ when a PISN blastwave overtakes a satellite halo with $M \sim 5 \times 10^{5} M_{\odot}$, triggering star formation there. At $z>10$, these enriched minihalos cause the Pop II SFR to be higher than this relation. Only after $z \sim 10$ does the SFR follows this exponential, which is a steeper time dependency than the $\exp (-z / 3)$ relation found in Hernquist \& Springel (2003), who also acknowledged that this behavior should occur at early times. At redshift 7 , it reaches $2 \times 10^{-2} \mathrm{M}_{\odot} \mathrm{yr}^{-1}$ $\mathrm{Mpc}^{-3}$. This value agrees with derived SFRs from $z=$ $6-10$ galaxy luminosity functions (e.g. Bouwens et al. 2011) and large-scale galaxy formation simulations (e.g. Schaye et al. 2010).

\subsection{Mass evolution}

Figure 4 a shows the total, metal-enriched stellar, and gas mass history of the most massive progenitors of both halos. The quiet halo undergoes a series of major mergers at $z>12$, growing by a factor of 30 to $2.5 \times 10^{7} M_{\odot}$ within 150 Myr. Subsequently, it only grows by a factor of 3 by $z=7$ mainly through smooth accretion from the filaments and IGM. It is the most massive halo in the simulation between redshifts 13 and 10. At the same time, the intense halo has a mass $M=3 \times 10^{6} M_{\odot}$, but it is contained in a biased region on a comoving scale of $50 \mathrm{kpc}$ with $\sim 25$ halos with $M \sim 10^{6} M_{\odot}$. After $z=10$, these halos hierarchically merge to form a $10^{9} M_{\odot}$ halo at $z=7$ with two major mergers at redshifts 10 and 7.9 , seen in the rapid increases in total mass in Figure 4. The merger history of the two halos are not atypical as dark matter halos can experience both quiescent and vigorous mass accretion rates.

Both halos start forming Pop II stars when $M=$ $10^{7} M_{\odot}$. This is consistent with the filtering mass $M_{f}$, which is plotted as a thick line in Figure 4a, of high-redshift halos when it accretes mainly from a pre-heated IGM (Gnedin \& Hui 1998; Gnedin 2000; Wise \& Abel 2008). Afterwards these halos can cool efficiently through $\mathrm{H}_{2}$ cooling, sustaining constant and sometimes bursting SF. The latter characteristics are equated with the definition of a galaxy. The quiet halo forms $10^{5} M_{\odot}$ of stars by $z=9$. This initial starburst photo-evaporates the majority of its molecular clouds, in addition to heating and ionizing the surrounding IGM out to a radius of $10-15 \mathrm{kpc}$ at $z=9$. These respectively reduce the in-situ and external cold gas supply that could feed future SF.

The gas fractions of both halos decrease from 0.15 to 0.08 by outflows driven by ionization fronts and blastwaves in their initial starbursts. The quiet halo does not have a major merger with any halo with $M>M_{f}$, leading to a small final gas fraction. These low-mass halos are photo-evaporated, hosting diffuse warm gas reservoirs instead of cold dense cores. After $z=10$, the halo mainly accretes warm diffuse gas from the filaments and IGM. In contrast, the intense halo grows from major mergers of halos with $M>M_{f}$. The progenitor halos involved in the major mergers are able to host molecular clouds and have higher gas fractions. Between $z=10$ and $z=8$, the gas fraction increases from 0.07 to 0.12 until it jumps to 0.14 when a gas-rich major merger occurs. The stellar mass accordingly increases with the ample supply of gas during this period.

\subsection{Metallicity evolution}

The evolution of the stellar and gas metallicity of both halos are illustrated in Figure $4 \mathrm{~b}$. PISNe from Pop III stars enrich the nearby IGM out to a radius of $10 \mathrm{kpc}$ and provides a metallicity floor of $\left[Z_{3} / H\right] \sim-3$. Ejecta from Pop II SNe initially enrich the ISM of both halos to an average $\left[\bar{Z}_{2} / H\right]$ between -3.5 and -3 .

In the quiet halo, an equilibrium of $\left[\bar{Z}_{2} / H\right] \sim-2.5$ is established between metal-rich outflows and metal-poor inflows. Galactic outflows are directed in the polar directions of the gas disk, keeping the adjacent filaments metal-poor. These features and a well-mixed ISM (cf. Wise \& Abel 2008; Greif et al. 2010) are apparent in the metallicity projections in Figure 2. The average stellar metallicity is within 0.5 dex of $\left[\bar{Z}_{2} / H\right]$.

In the intense halo, the first few Pop II star clusters have $[Z / H]$ between -1 and -2 and dominate the average stellar metallicity at $z>8$. The metallicity later increases by a factor of 30 to $\left[\bar{Z}_{2} / H\right]=-1.5$ through self-enrichment from a starburst. Because this halo is located in a large-scale overdensity, most of the ejecta falls back into the halo after reaching distances up to 20 comoving kpc, keeping the halo gas metallicity high because the inflows are relatively metal-rich themselves. After the $z \sim 8$ starburst, the average stellar metallicity follows the average gas metallicity within 0.1 dex.

To further demonstrate the metallicity floor in highredshift dwarf galaxies, we show the average metallicity from Pop III SNe $\left[\mathrm{Z}_{3} / \mathrm{H}\right]$ in all halos as a function of halo mass at $z=7,8,9$, and 10 in Fig. 5, and it is strongly dependent on halo mass. The $\left[\mathrm{Z}_{3} / \mathrm{H}\right]$ error bars 

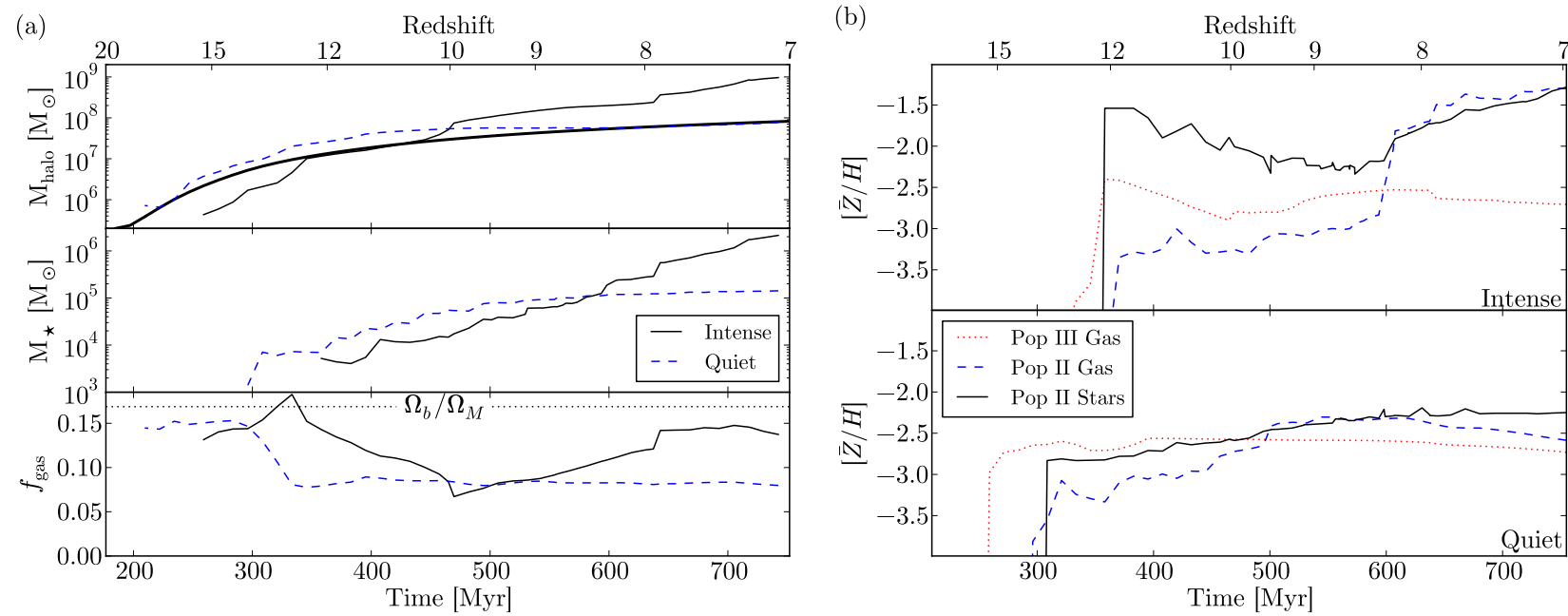

Fig. 4.- (a) Evolution of the total halo mass (top), stellar mass (middle), and gas fraction (bottom) of the quiet (dashed) and intense (solid) halos. In the top panel, the filtering halo mass is plotted as the thick black line (overlapping with the quiet halo after $z=9$. (b) Mass-weighted stellar metallicities and gas metallicities enriched by Pop II and Pop III SNe of the intense (top) and quiet (bottom) halos.

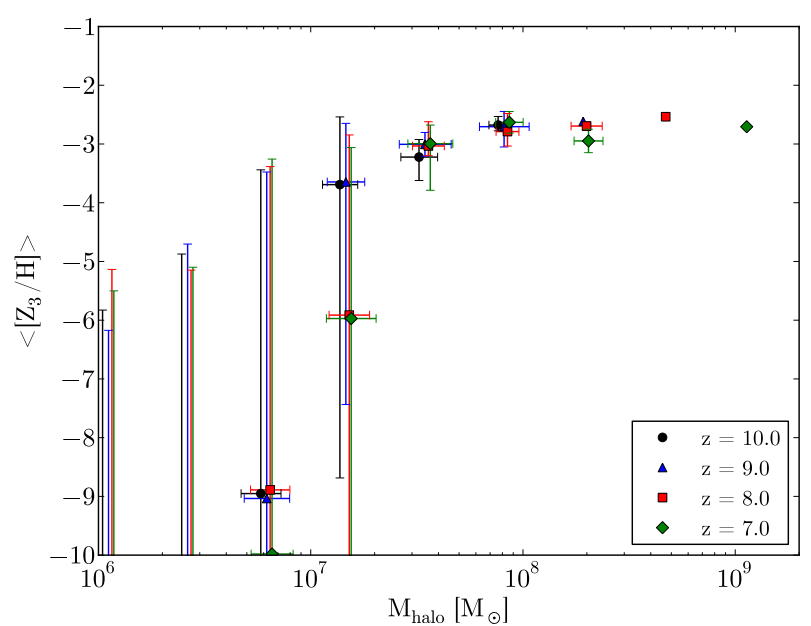

FIG. 5.- Average halo metallicity from Pop III SNe as a function of total halo mass. Inhomogeneous metal enrichment causes the large spread in metallicity at low masses. At $M_{\text {halo }}>3 \times 10^{7} M_{\odot}$, all halos are enriched by Pop III SNe above $[\mathrm{Z} / \mathrm{H}]>-4$. The $\mathrm{y}-$ axis error bars show the $25 \%$ and $75 \%$ percentile values because the underlying distribution is not Gaussian.

mark the $25 \%$ and $75 \%$ percentiles of the distribution at $M=M_{\text {halo }}$ because the underlying distribution is not necessarily Gaussian at all halo masses. Above $3 \times$ $10^{7} M_{\odot}$, all halos enriched to $\left[\mathrm{Z}_{3} / \mathrm{H}\right] \gtrsim-4$ with little time evolution, further illustrating the metallicity floor. Below this halo mass, the distribution is roughly bimodal composed of pristine and metal-enriched halos.

\subsection{Star formation history}

The most massive progenitor of the quiet halo interestingly never hosts a Pop III star. Instead a nearby halo forms a Pop III star, which (randomly) produces a PISNe at $z=16$. The blastwave overruns the most massive progenitor, and the dense core survives this event and is enriched by this PISN, triggering the transition to Pop II SF. Other progenitors host three Pop III stars, forming at $z=15.4,14.2,13.8$, with the latter producing a PISN. Metal enrichment from these two PISNe and Pop II SF quench Pop III SF in this halo. The progen- itors of the intense halo host a total of 56 Pop III stars with 21 producing PISNe and the other 35 producing intermediate-mass BHs. The first forms in a $6 \times 10^{5} M_{\odot}$ halo at $z=19$. Pop III stars form on a regular interval in the halo's progenitors until $z=9$ when most of these halos enter the metal-rich bubble surrounding the intense halo.

Figure 6 shows the SF history (SFH), metallicity distribution, and SF rates of both halos. A nearby PISNe provides a metallicity floor of $[\mathrm{Z} / \mathrm{H}]=-2.8$ in the quiet halo at which metallicity the first Pop II stars form. The stellar metallicity evolution exhibits what is expected from an isolated system with the stellar feedback steadily enriching the ISM, resulting in a correlation between stellar age and metallicity. After $z=10$ the metallicities plateau at $[\mathrm{Z} / \mathrm{H}]=-2.1$ for reasons previously discussed. The SFR peaks at $z=10$ and decreases as the cold gas reservoir is depleted. Around $z=7.5$, a 25:1 minor merger occurs, and the gas inside the satellite halo is compressed, triggering metal-poor, $[\mathrm{Z} / \mathrm{H}]=-3.2$, SF during its nearest approach. This halo remains metalpoor because most of metal enrichment in the quiet halo occurs in bi-polar flows perpendicular to the galaxy disk and filament. Stars with $[\mathrm{Z} / \mathrm{H}]<-3$ compose 1.6 percent of the total stellar mass.

In contrast with the quiet halo, the intense halo undergoes a few mergers of halos with an established stellar population. This creates a superposition of agemetallicity tracks in the $\mathrm{SFH}$, seen in the complexity of Figure 6. The first two Pop II stellar clusters have an unexpectedly high metallicity $[\mathrm{Z} / \mathrm{H}] \sim-1$, which occurs when a PISN blastwave triggers SF in two neighboring halos. Most of the early $\mathrm{SF}$ have $[\mathrm{Z} / \mathrm{H}]=-2.5$. At $z=9$, the halo's virial temperature reaches $10^{4} \mathrm{~K}$. This combined with a 10:1 merger creates a starburst that quickly enriches the halo to $[\mathrm{Z} / \mathrm{H}]=-1.5$ by $z=8$. The halo continues to enrich itself afterwards. The spikes in the scatter plot correspond to SN triggered SF in nearby molecular clouds that are enriched up to a factor of 10 with respect to the ISM. However their mass fraction are small compared to the total stellar mass. The starburst at $z=9$ creates a bimodal metallicity distribution with 

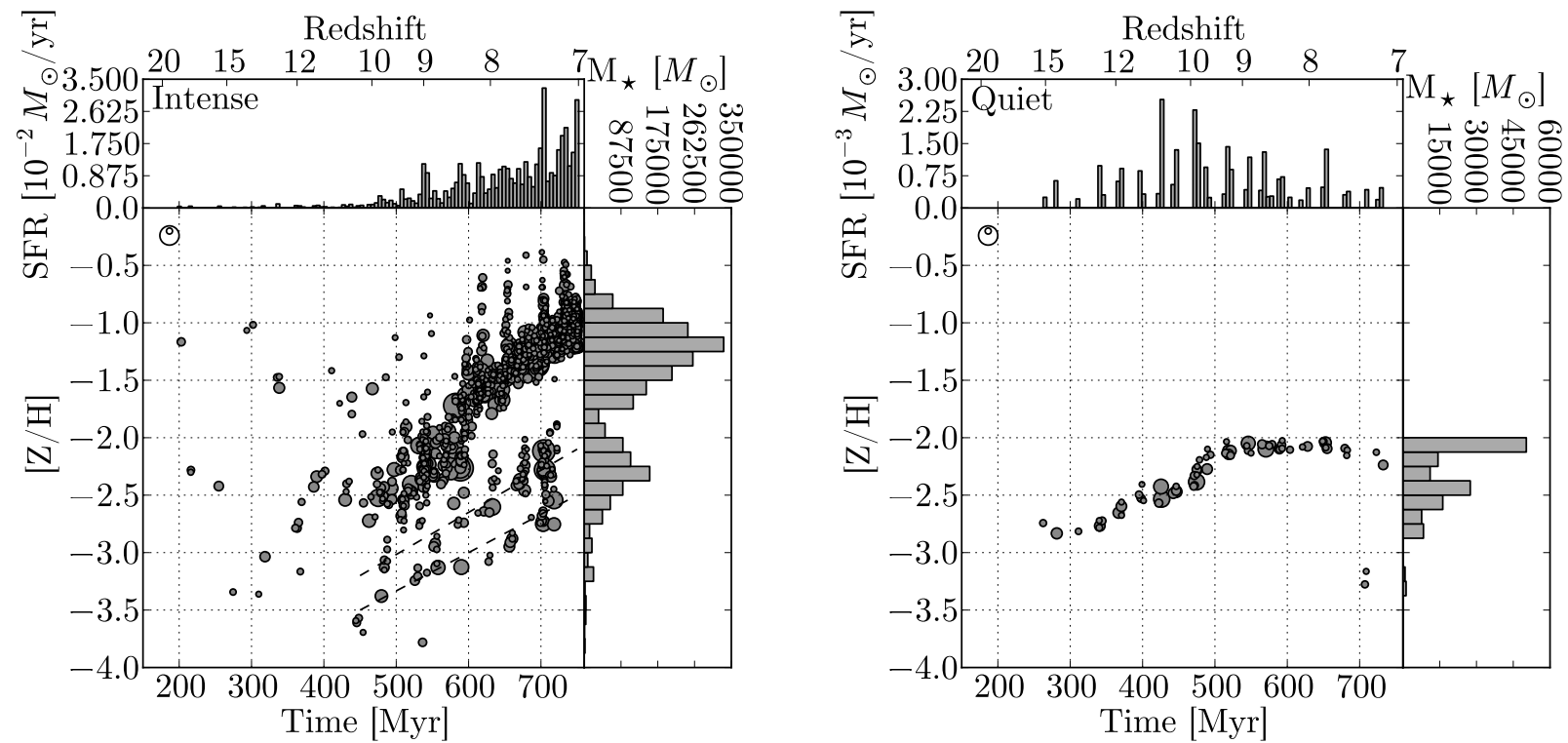

FIG. 6. - The scatter plots show the metal-enriched (Pop II) star formation history of the intense (left) and quiet (right) halos as a function of total metallicity, i.e. the sum of metal ejecta from both Pop II and Pop III SNe, at $z=7$. Each circle represents a star cluster, whose area is proportional to its mass. The open circles in the upper right represent $10^{3}$ and $10^{4} M_{\odot}$ star clusters. The upper histogram shows the SFR. The right histogram depicts the stellar metallicity distribution. The intense halo shows a large spread in metallicity at $z>10$ because these stars formed in progenitor halos that were enriched by different SN explosions. At $z<10$, the majority of stellar metallicities increase as the halo is self-enriched. The spikes in metallicity at $t=620,650$, and 700 Myr show induced star formation with enhanced metallicities in SN remnant shells. The dashed lines in the left panel guide the eye to two stellar populations that were formed in two satellite halos, merging at $z=7.5$. The quiet halo evolves in relative isolation and steadily increases its metallicity to $[\mathrm{Z} / \mathrm{H}] \sim-2$ until there is an equilibrium between in-situ star formation and metal-poor inflows from filaments.

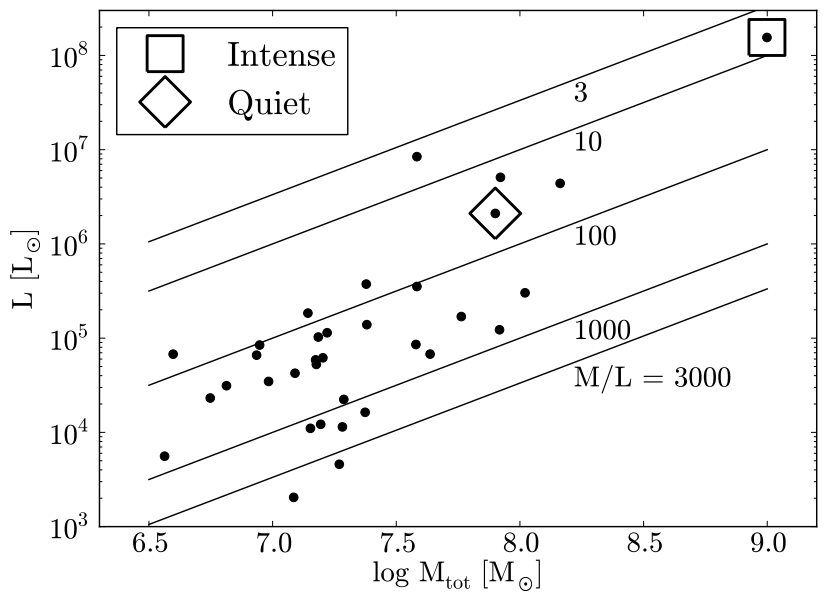

FIG. 7.- Total Pop II luminosities of halos versus total halo mass with constant mass-to-light ratios overplotted at redshift 7 . The halos with $M \gtrsim 10^{8} M_{\odot}$ form stars efficiently because they can cool through atomic line cooling, whereas the lower mass galaxies rely on molecular hydrogen cooling only. The low $\mathrm{M} / \mathrm{L}$ ratios and spread across two orders of magnitude are similar to ones observed in nearby ultra faint dwarf galaxies.

peaks at $[\mathrm{Z} / \mathrm{H}]=-2.4$ and -1.2 with the metal-rich component mainly being created after the starburst. Two systems with sizable stellar components merge into the halo at $z \sim 8$, and their stellar populations, which are traced by dashed lines in Figure 6, are still discernible in the metallicity-age plot. Stars with $[\mathrm{Z} / \mathrm{H}]<-3$ compose 1.8 percent of the total stellar mass.

\subsection{Mass-to-light ratios}

Mass-to-light ratios are crucial in interpreting observations when connecting the galaxy to its DM halo, and ultimately to models of galaxy formation. For this paper, we use a simple model to calculate the total stellar luminosity of each galaxy. In a later paper, we will follow up with more sophisticated models to determine more accurate observables. Stars in each particle are treated as an instantaneous burst. Using the empirical law of main sequence lifetime $t_{\mathrm{MS}} \propto M^{-5 / 2}$, the maximum stellar mass in such a system is dependent only on its age $t_{\star}$, so $M_{\text {up }}=\left(t_{\star} / 10 \text { Gyr }\right)^{-2 / 5}$. Then combining another scaling law $L \propto M^{7 / 2}$ and the Salpeter IMF $\psi(M) \propto M^{-\alpha}$,

$$
\begin{aligned}
L / M_{\odot} & =\int_{M_{\mathrm{lo}}}^{M_{\mathrm{up}}} \psi(M) L(M) d M \\
& \simeq M_{\mathrm{up}}^{9 / 2-\alpha}(9 / 2-\alpha)^{-1},
\end{aligned}
$$

where we set $M_{\mathrm{lo}} \equiv 0$ because the total luminosity of low-mass stars is negligible. Then the galaxy luminosity is the sum of all star particle luminosities, which we plot in Fig. 7 with respect to halo mass, along with the DM halo mass function.

Recall that the most massive halo $\left(M_{\text {tot }}=10^{9} M_{\odot}\right)$ is undergoing a starburst at $z=7$, translating into a relatively high $M / L=6$. The lower mass galaxies show a spread in M/L between 30 and 3000, similar to the Sloan Digital Sky Survey (SDSS) observations of local ultra faint dwarf $(\mathrm{uFd})$ galaxies (e.g. Strigari et al. 2008). It has been suggested that these low-mass $\left(M_{\text {tot }} \lesssim 10^{8} M_{\odot}\right)$ galaxies that cannot cool through hydrogen line cooling are the progenitors of $\mathrm{uFd}$ galaxies (Bovill \& Ricotti 2011a,b), where all of the star formation occurs before reionization and was subsequently terminated by UV heating from external sources. These galaxies have stel- 
lar masses ranging from $210 M_{\odot}$ (SDSS J1058+2843) to $3.2 \times 10^{5} M_{\odot}$ (Dra) with most between $10^{3}$ and $10^{4} M_{\odot}$. (Martin et al.|2008). Because the total stellar population would be sampled, on average, by several star particles, we cannot make robust conclusions about the nature of $\mathrm{uFd}$ galaxies.

\section{DISCUSSION AND CONCLUSIONS}

We have focused on the birth of two galaxies prior to reionization with a cosmological AMR radiation hydrodynamics simulation with very high spatial (1 pc comoving) and mass resolution $\left(1800 M_{\odot}\right)$. With this resolution, we are able to follow the Pop III star formation and radiative and chemical feedback effects in hundreds of minihalos that contribute to the formation of a protogalaxy. Supernovae from Pop III stars provide the necessary heavy elements for the transition to a Population II stellar population, which we have directly simulated. The galaxies simulated here represent a population of uncharacterized high-redshift dwarf galaxies, which should be detected with the upcoming James Webb Space Telescope, that should contribute a significant fraction of the ionizing photons during reionization. Recently, a $z=8.6$ spectroscopically confirmed galaxy was found to reside in an $\mathrm{H}$ II region that extends beyond $1 \mathrm{Mpc}$. However, using the SFRs and Ly $\alpha$ continuum as estimators, this galaxy produces enough ionizing radiation to create an $\mathrm{H}$ II region between $\sim 0.1\left(f_{\text {esc }} / 0.1\right)^{1 / 3} \mathrm{Mpc}$ and $0.5\left(f_{\text {esc }} / 0.1\right)^{1 / 3} \mathrm{Mpc}$, where $f_{\text {esc }}$ is the fraction of ionizing radiation escaping into the IGM (Lehnert et al. 2010). This discrepancy could be caused by undetected satellite dwarf galaxies, such ones presented here, that contribute to local radiation field, or an ionizing radiation escape fraction near unity (Wise \& Cen 2009; Razoumov \& Sommer-Larsen 2010; Paardekooper et al. 2011; Yajima et al. 2011).

We find that one PISN is sufficient to enrich the starforming halo and surrounding $\sim 5 \mathrm{kpc}$ to a metallicity of $10^{-3} Z_{\odot}$, given $M_{\text {char }}=100 M_{\odot}$. DLA systems have a metallicity floor on the same order (Wolfe et al. 2005; Penprase et al. 2010), and metal enrichment from Pop III SNe provides a possible explanation (Kobavashi et al. 2011). Our simulation strengthens this claim of a metallicity floor from Pop III SNe (see also Tornatore et al. 2007; Karlsson et al. 2008; (Maio et al. 2011), where our work improves on previous studies by resolving starforming molecular clouds and employing more realistic feedback physics, in particular, radiation transport.

If the first stars have a lower characteristic mass that favor hypernovae (Tumlinson 2007), then this metallicity floor should be lowered by a factor of $\sim 10$ because the metal ejecta from a $40 M_{\odot}$ hypernova is 8.6 $M_{\odot}$ (Nomoto et al. 2006), compared to $85 M_{\odot}$ of metals produced by a $180 M_{\odot}$ PISN (Heger \& Woosley 2002b). Both explosions have approximately $3 \times 10^{52} \mathrm{erg}$. Therefore the blastwaves from these two explosions should expand to equal radii before stalling in the IGM. We note that stronger metal-line cooling in the PISN case may cause the blastwave to stall at a marginally smaller radius.

Building upon this idea of a lower metallicity floor, consider a pre-galactic halo that has hosted a Pop III star, producing a SN. The majority of the gas is ejected into the IGM and re-accretes after $\sim 10-50 \mathrm{Myr}$ (Johnson et al. 2007; Wise \& Abel 2008). What happens if the metallicity is not greater than the critical metallicity? Massive Pop III stars would form again in this halo; however, enriching the surrounding IGM with Pop III SNe and ejecting material becomes more difficult in halos with $M \gtrsim 10^{7} M_{\odot}$ (Whalen et al. 2008). Thus it is more likely for these halos to retain more of its gas during star formation episodes and self-enrich its host halo and less of the IGM. This would accelerate the transition from Pop III to II in more massive halos.

We conclude that it only takes one, at most two, $\mathrm{SNe}$ from Pop III stars in the halo progenitors to complete the transition to Population II (Frebel \& Bromm 2010). However, this does not prohibit more than two instances of Pop III star formation in halo from happening because the stars can produce $\mathrm{BHs}$ with little or no metal ejecta, keeping the surrounding environment pristine. Even though we find that Pop III SNe create a floor of $\sim 10^{-3} \mathrm{Z}_{\odot}$, gas with lower metallicities can still exists in nearby halos, where the metal-rich SN ejecta will mix slowly into dense cores as the blastwave overtakes them (Cen \& Riquelme 2008). These environments may be the host of true extremely metal-poor star formation, such as the recently discovered halo star with all detected metals below $10^{-4.2} \mathrm{Z}_{\odot}$ (Caffau et al. 2011).

We also find that the merger history plays an important role in supplying gas into halos after its first epoch of SF. Mergers of halos below the filtering mass are inefficient in providing gas whereas the opposite is true for merging halos above the filtering mass. Halos do not necessarily need $T_{\mathrm{vir}} \geq 10^{4} \mathrm{~K}$ to form significant stellar populations; however SFRs dramatically increase, and thus metal enrichment, when this threshold is reached. Halos with mostly gas-poor mergers or a quiescent merger history result in a monotonic increase in metallicity with time. SFHs become more complex with multiple metallicity-age tracks if the halo experiences mergers with other halos that have an established stellar population. Furthermore SF that is triggered by blastwaves interacting with molecular clouds can have metallicities up to a factor of 10 higher than the main starburst. Starbursts at $T_{\mathrm{vir}}=10^{4} \mathrm{~K}$ can enrich the host halo enough to create a bimodal metallicity distribution, where the metal-poor component is created before the burst. Note that mergers of stellar populations can also create a similar bimodal distribution, which are observed in dSphs (Battaglia et al. 2011).

Because our simulation only samples 1 comoving $\mathrm{Mpc}^{3}$, it misses the more rare density fluctuations and is biased toward late galaxy formation (Barkana \& Loeb 2004). In such rare galaxies, the physical scenario described by this work is largely unchanged but is shifted to higher redshifts. Thus our results can be generalized to any epoch prior to cosmic reionization. However, two possible consequences in very high $(z \gtrsim 20)$ redshift galaxy formation are 1) an intermediate mass IMF (Larson 1998; Tumlinson 2007; Smith et al. 2009), resulting from the CMB temperature floor, could be more prevalent, and 2) streaming gas velocities arising from the recombination epoch (Tseliakhovich \& Hirata 2010) could suppress star formation in halos below $10^{6} M_{\odot}$ (Tseliakhovich et al. 2011; Greif et al. 2011b). 
We have shown that it is possible to simulate the formation of a high-redshift dwarf galaxy and its entire SFH with radiative and mechanical feedback. These results provide invaluable insight on the first galaxies and the role of metal-free stars in the early universe. We plan to follow up this work by focusing on observational connections with high-redshift galaxies and Local Group dwarf galaxies in the near future that can better constrain the physical models and assumptions, such as the critical metallicity, radiation backgrounds, and IMFs, used in $a b$ initio dwarf galaxy simulations.

JHW thanks Renyue Cen, Amina Helmi, Marco Spaans, and Eline Tolstoy for enlightening discussions. We thank an anonymous referee for helpful suggestions. JHW is partially supported by NASA through Hubble Fellowship grant \#120-6370 awarded by the Space
Telescope Science Institute, which is operated by the Association of Universities for Research in Astronomy, Inc., for NASA, under contract NAS 5-26555. MJT and MLN acknowledge partial support from NASA ATFP grant NNX08AH26G. MJT acknowledges support by the NSF CI TraCS fellowship award OCI-1048505. Computational resources were provided by NASA/NCCS award SMD-09-1439. TA acknowledges financial support from the Baden-Württemberg-Stiftung under grant P-LSSPII/18, the Heidelberg Institut für Theoretische Studien. This work was partially supported by NASA ATFP grant NNX08AH26G, NSF AST-0807312, and NSF AST1109243. This research has made use of NASAs Astrophysics Data System Bibliographic Services. The majority of the analysis and plots were done with yt (Turk et al. 2011).

\section{REFERENCES}

Abel, T., Anninos, P., Zhang, Y., \& Norman, M. L. 1997, New Astronomy, 2, 181

Abel, T., Bryan, G. L., \& Norman, M. L. 2002, Science, 295, 93

Abel, T., \& Wandelt, B. D. 2002, MNRAS, 330, L53

Abel, T., Wise, J. H., \& Bryan, G. L. 2007, ApJ, 659, L87

Anninos, P., Zhang, Y., Abel, T., \& Norman, M. L. 1997, New Astronomy, 2, 209

Barkana, R., \& Loeb, A. 2004, ApJ, 609, 474

Battaglia, G., Tolstoy, E., Helmi, A., Irwin, M., Parisi, P., Hill, V., \& Jablonka, P. 2011, MNRAS, 411, 1013

Beers, T. C., \& Christlieb, N. 2005, ARA\&A, 43, 531

Bertschinger, E. 2001, ApJS, 137, 1

Bouwens, R.' J., Illingworth, G. D., Oesch, P. A., Labbé, I., Trenti, M., van Dokkum, P., Franx, M., Stiavelli, M., Carollo,

C. M., Magee, D., \& Gonzalez, V. 2011, ApJ, 737, 90

Bovill, M. S., \& Ricotti, M. 2011a, ApJ, 741, 17

. 2011b, ApJ, 741, 18

Bromm, V., Coppi, P. S., \& Larson, R. B. 2002, ApJ, 564, 23

Bromm, V., Ferrara, A., Coppi, P. S., \& Larson, R. B. 2001, MNRAS, 328, 969

Bryan, G. L., \& Norman, M. L. 1997, ArXiv Astrophysics e-prints Bryan, G. L., Norman, M. L., Stone, J. M., Cen, R., \& Ostriker, J. P. 1995, Computer Physics Communications, 89, 149

Caffau, E., Bonifacio, P., François, P., Sbordone, L., Monaco, L., Spite, M., Spite, F., Ludwig, H.-G., Cayrel, R., Zaggia, S., Hammer, F., Randich, S., Molaro, P., \& Hill, V. 2011, Nature, 477,67

Cen, R., \& Ostriker, J. P. 1992, ApJ, 399, L113

Cen, R., \& Riquelme, M. A. 2008, ApJ, 674, 644

Chabrier, G. 2003, PASP, 115, 763

Clark, P. C., Glover, S. C. O., Bonnell, I. A., \& Klessen, R. S. 2009, ArXiv e-prints (0904.3302)

Clark, P. C., Glover, S. C. O., \& Klessen, R. S. 2008, ApJ, 672, 757

Clark, P. C., Glover, S. C. O., Smith, R. J., Greif, T. H., Klessen, R. S., \& Bromm, V. 2011, Science, 331, 1040

Couchman, H. M. P. 1991, ApJ, 368, L23

Efstathiou, G., Davis, M., White, S. D. M., \& Frenk, C. S. 1985 , ApJS, 57, 241

Ferrara, A. 1998, ApJ, 499, L17+

Frebel, A., \& Bromm, V. 2010, ArXiv e-prints (1010.1261)

Frebel, A., Kirby, E. N., \& Simon, J. D. 2010, Nature, 464, 72

Furlanetto, S. R., \& Loeb, A. 2005, ApJ, 634, 1

Glover, S. C. O., \& Abel, T. 2008, MNRAS, 388, 1627

Glover, S. C. O., \& Mac Low, M.-M. 2007, ApJS, 169, 239

Gnedin, N. Y. 2000, ApJ, 542, 535

Gnedin, N. Y., \& Hui, L. 1998, MNRAS, 296, 44

Górski, K. M., Hivon, E., Banday, A. J., Wandelt, B. D., Hansen F. K., Reinecke, M., \& Bartelmann, M. 2005, ApJ, 622, 759

Greif, T. H., Glover, S. C. O., Bromm, V., \& Klessen, R. S. 2010, ApJ, 716, 510

Greif, T. H., Johnson, J. L., Bromm, V., \& Klessen, R. S. 2007, ApJ, 670, 1

Greif, T. H., Springel, V., White, S. D. M., Glover, S. C. O. Clark, P. C., Smith, R. J., Klessen, R. S., \& Bromm, V. 2011a, ApJ, 737, 75

Greif, T. H., White, S. D. M., Klessen, R. S., \& Springel, V. 2011b, ApJ, 736, 147

Heger, A., Fryer, C. L., Woosley, S. E., Langer, N., \& Hartmann, D. H. 2003, ApJ, 591, 288
Heger, A., \& Woosley, S. E. 2002a, ApJ, 567, 532

-. 2002b, ApJ, 567, 532

Hernquist, L., \& Springel, V. 2003, MNRAS, 341, 1253

Johnson, J. L., Greif, T. H., \& Bromm, V. 2007, ApJ, 665, 85

Karlsson, T., Johnson, J. L., \& Bromm, V. 2008, ApJ, 679, 6

Kitayama, T., Yoshida, N., Susa, H., \& Umemura, M. 2004, ApJ, 613,631

Kobayashi, C., Tominaga, N., \& Nomoto, K. 2011, ApJ, 730, L14+

Komatsu, E., et al. 2011, ApJS, 192, 18

Komiya, Y., Habe, A., Suda, T., \& Fujimoto, M. Y. 2010, ApJ, 717,542

Krumholz, M. R., \& McKee, C. F. 2005, ApJ, 630, 250

Larson, R. B. 1998, MNRAS, 301, 569

Lehnert, M. D., Nesvadba, N. P. H., Cuby, J., Swinbank, A. M., Morris, S., Clément, B., Evans, C. J., Bremer, M. N., \& Basa, S. 2010 , Nature, 467,940

Machacek, M. E., Bryan, G. L., \& Abel, T. 2001, ApJ, 548, 509

Maio, U., Ciardi, B., Dolag, K., Tornatore, L., \& Khochfar, S. 2010, MNRAS, 407, 1003

Maio, U., Khochfar, S., Johnson, J. L., \& Ciardi, B. 2011, MNRAS, 414, 1145

Martin, N. F., de Jong, J. T. A., \& Rix, H.-W. 2008, ApJ, 684, 1075

Matsumoto, M., \& Nishimura, T. 1998, ACM Trans. Model. Comput. Simul., 8, 3

Nomoto, K., Tominaga, N., Umeda, H., Kobayashi, C., \& Maeda, K. 2006, Nuclear Physics A, 777, 424

Omukai, K., Tsuribe, T., Schneider, R., \& Ferrara, A. 2005, ApJ, 626,627

O'Shea, B. W., Bryan, G., Bordner, J., Norman, M. L., Abel, T., Harkness. R.. \& Kritsuk, A. 2004, ArXiv Astrophysics e-prints (astro-ph/0403044)

O'Shea, B. W., \& Norman, M. L. 2007, ApJ, 654, 66

. 2008, ApJ, 673, 14

Paardekooper, J.-P., Pelupessy, F. I., Altay, G., \& Kruip, C. J. H. 2011, A\&A, 530, A87

Penprase, B. E., Prochaska, J. X., Sargent, W. L. W. Toro-Martinez, I., \& Beeler, D. J. 2010, ApJ, 721, 1

Razoumov, A. O., \& Sommer-Larsen, J. 2010, ApJ, 710, 1239

Ricotti, M., Gnedin, N. Y., \& Shull, J. M. 2008, ApJ, 685, 21

Safranek-Shrader, C., Bromm, V., \& Milosavljević, M. 2010, ApJ, 723,1568

Salvadori, S., Schneider, R., \& Ferrara, A. 2007, MNRAS, 381, 647

Scannapieco, E., Schneider, R., \& Ferrara, A. 2003, ApJ, 589, 35 Schaerer, D. 2002, A\&A, 382, 28

-. 2003, A\&A, 397, 527

Schaye, J., Dalla Vecchia, C., Booth, C. M., Wiersma, R. P. C., Theuns, T., Haas, M. R., Bertone, S., Duffy, A. R., McCarthy, I. G.. \& van de Voort, F. 2010, MNRAS, 402, 1536

Schneider, R., Omukai, K., Inoue, A. K., \& Ferrara, A. 2006, MNRAS, 369, 1437

Smith, B., Sigurdsson, S., \& Abel, T. 2008, MNRAS, 385, 1443

Smith, B. D., Hallman, E. J., Shull, J. M., \& O'Shea, B. W. 2011 , ApJ, 731, 6

Smith, B. D., Turk, M. J., Sigurdsson, S., O'Shea, B. W., \&

Norman, M. L. 2009, ApJ, 691, 441

Springel, V., \& Hernquist, L. 2003, MNRAS, 339, 289

Stacy, A., Greif, T. H., \& Bromm, V. 2010, MNRAS, 403, 45 
Strigari, L. E., Bullock, J. S., Kaplinghat, M., Simon, J. D., Geha, M., Willman, B., \& Walker, M. G. 2008, Nature, 454, 1096

Tafelmeyer, M., Jablonka, P., Hill, V., Shetrone, M., Tolstoy, E., Irwin, M. J., Battaglia, G., Helmi, A., Starkenburg, E., Venn, \& Szeifert, T. 2010, A\&A, 524, A58+

Tan, J. C., Krumholz, M. R., \& McKee, C. F. 2006, ApJ, 641, L121

Tornatore, L., Ferrara, A., \& Schneider, R. 2007, MNRAS, 382, 945

Toro, E. F., Spruce, M., \& Speares, W. 1994, Shock Waves, 4, 25, 10.1007/BF01414629

Trenti, M., \& Shull, J. M. 2010, ApJ, 712, 435

Trenti, M., Stiavelli, M., \& Shull, J. M. 2009, ApJ, 700, 1672

Truelove, J. K., Klein, R. I., McKee, C. F., Holliman, II, J. H., Howell, L. H., \& Greenough, J. A. 1997, ApJ, 489, L179+

Tseliakhovich, D., Barkana, R., \& Hirata, C. M. 2011, MNRAS, 1501

Tseliakhovich, D., \& Hirata, C. 2010, Phys. Rev. D, 82, 083520

Tumlinson, J. 2006, ApJ, 641, 1

-. 2007, ApJ, 664, L63

-. 2010, ApJ, 708, 1398

Turk, M. J., Ábel, T., \& O'Shea, B. 2009, Science, 325, 601

Turk, M. J., Smith, B. D., Oishi, J. S., Skory, S., Skillman, S. W., Abel, T., \& Norman, M. L. 2011, ApJS, 192, 9
Whalen, D., Abel, T., \& Norman, M. L. 2004, ApJ, 610, 14 Whalen, D., van Veelen, B., O'Shea, B. W., \& Norman, M. L. 2008, ApJ, 682, 49

Wise, J. H., \& Abel, T. 2007, ApJ, 665, 899

- 2008, ApJ, 685, 40

-. 2011, MNRAS, 414, 3458

Wise, J. H., \& Cen, R. 2009, ApJ, 693, 984

Wolcott-Green, J., Haiman, Z., \& Bryan, G. L. 2011, MNRAS, 1673

Wolfe, A. M., Gawiser, E., \& Prochaska, J. X. 2005, ARA\&A, 43, 861

Woodward, P., \& Colella, P. 1984, Journal of Computational Physics, 54, 115

Woosley, S. E., \& Weaver, T. A. 1995, ApJS, 101, 181

Yajima, H., Choi, J.-H., \& Nagamine, K. 2011, MNRAS, 412, 411

Yoshida, N., Abel, T., Hernquist, L., \& Sugiyama, N. 2003, ApJ, 592,645

Yoshida, N., Bromm, V., \& Hernquist, L. 2004, ApJ, 605, 579

Yoshida, N., Oh, S. P., Kitayama, T., \& Hernquist, L. 2007, ApJ, 663,687

Yoshida, N., Omukai, K., \& Hernquist, L. 2008, Science, 321, 669

Zahn, O., Mesinger, A., McQuinn, M., Trac, H., Cen, R., \& Hernquist, L. E. 2011, MNRAS, 414, 727 